\title{
ESTUDIO BIBLIOMETRICO SOBRE DEPRESION Y SUICIDIO EN NIÑOS, NIÑAS Y ADOLESCENTES, PERIODO 2003-2014
}

\section{BIBLIOMETRIC STUDY ON DEPRESSION AND SUICIDE IN CHILDREN AND ADOLESCENTS, 2003-2014 PERIOD}

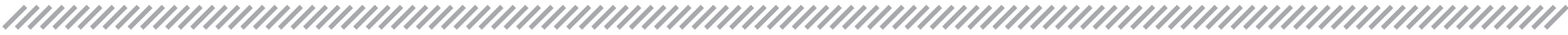

AUTORES: Jesús Peña Mercedes ${ }^{1}$, Loo Martínez Lucia², Camargo Herrera Luis ${ }^{3}$, Castro Hurtado Verónica ${ }^{4}$, Cadenas Huiza Henry ${ }^{5}$, Cabello Collachagua Yaneth ${ }^{6}$, Daga León Eliana ${ }^{7}$, Flores Ventocilla Diana ${ }^{8}$, Huaranga Paitan Leydi $i^{9}$ León Carlos Karol ${ }^{10}$, Mallqui Cuadros Ana ${ }^{11}$, Naupari Gutiérrez Lucia ${ }^{12}$, Ochoa Quiñones Katherin ${ }^{13}$, Sánchez Jesús Carla ${ }^{14}$, Vera Prado Vianca ${ }^{15}$, Urco Cáceres Sandro ${ }^{16}$
\end{abstract}

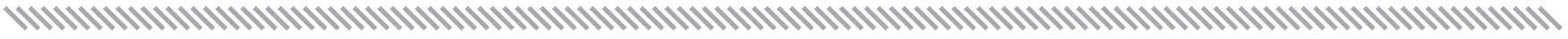

\section{RESUMEN}

La depresión y suicidio en nuestros niños, niñas y adolescentes es un problema de salud pública que preocupa a la sociedad en general, las proyecciones de la Organización Mundial de la Salud, sobre la salud mental en la población infanto-juvenil no son alentadoras por ello, considerando que es importante conocer el estado de la producción científica en este tema y ser un referente para posteriores investigaciones,

OBJETIVO: Analizar la producción científica publicada en depresión y suicido en niños, niñas y adolescentes del 2003 al 2014 a través del análisis bibliométrico de 94 artículos accedidos de las principales bases de datos, en lengua española. Nuestros hallazgos son, que los temas más investigados son depresión en adolescentes $(23.4 \%)$ seguidos de depresión en niños $(21.2 \%)$, siendo Colombia (26.6\%), México (17.02\%) y España $(15.9 \%)$ los que más han publicado. la producción científica empieza a incrementarse a partir del año 2012.

METODO: Estas investigaciones son del tipo aplicada-sustantiva $(63.9 \%)$, en las que sobresale el Diseño descriptivo correlacional (22.4\%) y el descriptivo simple (16.4\%). Las muestras suelen ser del tipo no probabilístico intencional $(65.7 \%)$ siendo el tamaño mayoritario de 101 y 500 participantes $(40.3 \%)$, en ellas se han incluido varones y mujeres $(95.5 \%)$, siendo los adolescentes del nivel secundario los más investigados (73\%), el 88\% no reporta nivel socioeconómico. Entre los instrumentos de medición se reportan los cuestionarios y escalas y tests psicométricos (71\%) siendo el Cuestionario de depresión para niños CDS el más utilizado. Finalmente, más del $84 \%$ de producción corres- ponde a autorías múltiples.

PALABRAS CLAVE: Análisis bibliométrico, depresión niños, depresión adolescentes, suicidio niños, suicidio adolescentes.

\begin{abstract}
Depression and suicide in our children and adolescents is a public health problem that concerns society as a whole, the projections of the World Health Organization on mental health in the child population are not encouraging for it Whereas it is important to know the state of scientific production in this area and become a reference for further research,
\end{abstract}

OBJECTIVE: To analyze the scientific production published in depression and suicide in children and adolescents from 2003 to 2014 through the bibliometric analysis of 94 major items accessed databases, in Spanish. Our findings are that the issues are investigated adolescent depression (23.4\%) followed by depression in children (21.2\%), and Colombia (26.6\%), Mexico $(17.02 \%)$ and Spain (15.9\%) who have most published . scientific production begins to increase from 2012.

METHOD: This research is applied-substantive type (63.9\%), where stands the descriptive correlational design (22.4\%) and the simple descriptive (16.4\%). Samples are usually non-probabilistic intentional (65.7\%) being the major size of 101 and 500 participants $(40.3 \%)$ of them have been included men and women $(95.5 \%)$, while teenagers investigated the secondary level ( $73 \%$ ), 88\% reported no socioeconomic status. Among the measuring instruments and questionnaires and psychometric scales $(71 \%)$ tests are reported to be

'Docente investigadora de la Universidad Peruana Los Andes. Asesora del Semillero de Investigación

${ }_{2}^{2}$ Psicóloga Hospital Domingo Olavegoya-Jauja. Co-Asesora del Semillero de Investigación

$345678,10111_{12}^{13} 14,{ }_{15}$ Estudiantes de Psicología de la UPLA. Integrantes del Semillero de Investigación

${ }^{16}$ Docente de la Escuela de Psicología-UPLA. Integrante del Semillero de Investigación 
the children's depression questionnaire used the CDS. Finally, over $84 \%$ of production is for multiple authorship.

KEYWORDS: bibliometric analysis, depressed children, adolescents depression, suicidal children, adolescents suicide.

\section{INTRODUCCION}

Para la Organización Panamericana de la Salud (OPS), los trastornos mentales se consideran un problema de salud en casi todo el mundo y se calcula que para el año 2020 la depresión será la segunda dolencia más frecuente y la primera causa de ausentismo laboral y escolar en países desarrollados, La depresión, según la Organización Mundial de la Salud (OMS) es una de las tres primeras causas de enfermedad y discapacidad entre los jóvenes de ambos sexos, Las cifras hablan de un 20 por ciento de los niños y adolescentes del planeta con alguna enfermedad mental, pero los síntomas depresivos y la depresión se han convertido en un problema de magnitud notoria.. El trastorno depresivo mayor en niños y adolescentes es una enfermedad frecuente con una prevalencia de 0,4 a 2,5 por ciento en niños, y de 0,4 a 8,3 por ciento en adolescentes.

Se considera que es la segunda patología más vista en psiquiatría infantil y se asocia con disfunción familiar, escolar y conducta suicida". 1

La depresión es una dolencia afectiva que por su aparición en la infancia sigue un curso crónico y puede desarrollar consecuencias secundarias como déficits psicosociales, alcoholismo, abuso de sustancias y personalidad antisocial, bajo rendimiento académico, intentos de suicidio y suicidio. La conducta suicida en niños y adolescentes en la actualidad es considerada como un problema de salud pública relevante, pues ocupa un lugar entre las primeras 10 causas de muerte en las estadísticas de la Organización Mundial de la Salud. Se considera que cada día se suicidan en el mundo al menos 1.110 personas y lo intentan cientos de miles; independientemente de la geografía, cultura, etnia, religión, posición socioeconómica, entre otras. El suicidio al igual que el intento de suicidio es poco estudiado en niños y adolescentes. 1

El fenómeno del suicidio de niños y adolescentes tiene características cuantitativas y cualitativas propias, diferentes del suicidio de los adultos, lo que hace necesario considerar el impacto del desarrollo en la conducta suicida. El suicidio es todo acto por el cual un sujeto se autoinflinge la muerte; muchas veces se considera que las personas que cometen este acto tienen algún trastorno mental, pero no en todos los casos es así; en ciertas ocasiones el suicidio es una expresión de un estado afectivo de angustia y desesperación, por lo general se relaciona con estados de ánimo depresivos y otros factores de riesgo2

El estudio del suicidio se hace relevante en tanto que es un fenómeno que afecta a toda una sociedad, sin discriminación de sexo, edad, clase social, ocupación y demás aspectos psicosociales. Actualmente, la conducta suicida es considerada como un hecho de causas multifactoriales en la que intervienen elementos biológicos, psicológicos, sociales y ambientales. La literatura sobre el suicidio en adultos es abundante así como los datos descriptivos y epidemiológicos al respecto.

A decir de Méndez, afortunadamente la investigación sobre depresión infantil y del adolescente ha progresado notablemente en los últimos 20 años, aunque los logros han sido desiguales, proliferando los estudios epidemiológicos y de validación de instrumentos de evaluación en comparación con los de desarrollo de protocolos terapéuticos y de valoración de tratamientos.

Resulta necesario medir el crecimiento de la producción científica relacionada con la depresión y suicidio en niños, niñas y adolescentes desde un estudio bibliométrico, el cual mide el crecimiento de la ciencia por medio de la producción de literatura científica aplicando métodos estadísticos, para poder establecer el desarrollo de una disciplina. Este análisis se efectúa por medio de las referencias bibliográficas de las publicaciones tomando como referencia un periodo de tiempo determinado.

Los antecedentes más resaltantes asociados a estudios bibliométricos en depresión infantil o adolescentes los encontramos:

En el 2003, Diez, M reportó los resultados del Análisis bibliométrico sobre depresión infantil en España desde 1875 hasta junio de 2002. El número de trabajos encontrados fue de 208 , de los cuales el $88 \%$ ha sido publicado en las dos últimas décadas (1983 a 2002), mientras que en los restantes 107 años, desde 1875 hasta 1982, sólo el $12 \%$. Destaca a Aquilino Polaino como gran productor con 34 publicaciones, seguido de Doménech Llobeira, Victoria del Barrio y Conde López. Ballesteros Alcalde tiene el índice de colaboración más alto con una puntuación de 1,88, mientras que Josep Toro presenta un índice de colaboración cero. El número de 
trabajos firmados por un autor fue de 105, es decir, el $50,48 \%$. El contenido de los trabajos es fundamentalmente teórico, un $54 \%$, y de éstos un $18 \%$ se refiere al diagnóstico y clasificación de la depresión infantil y tan solo el $8 \%$ el estudio de factores de riesgo y prevención. 3

Palacios-Espinosa, X.; Barrera, A, y Ordóñez, M. Y Peña, M, comunican los resultados del Análisis bibliométrico de la producción científica sobre suicidio en niños en el período 1985-2005. Las bases de datos consultados fueron: Lilacs, Medline, Ebsco Host y Ovid. El número de artículos revisados fue 84, siendo Estados Unidos el país de mayor producción con un $65,85 \%$ de los trabajos publicados. Casi el total de los artículos se encuentran firmados por uno, dos o tres autores; los autores más productivos son Shaffer, King, Kazdin, Jiang, Rosenbaum, Bridge, Axelson, Brent, Ryan y Mann cada uno con dos trabajos publicados y Pfeffer con un total de cuatro artículos. Los contenidos más comunes son los de revisión teórica del suicidio en niños (conceptualización, suicidio consumado, ideación suicida e intención suicida) y evaluación de suicidio en esta población. 4

Herrera, Eduar.; Núñez, A.Tobón, S.;Arias, D, el año 2009, publican los hallazgos de un análisis bibliométrico referente a los trabajos sobre la depresión infantil, publicados desde 1985 hasta el año 2006. Utilizaron la base de datos Hinari de la Organización Mundial de la Salud (OMS) y Psicodoc, donde se ubican investigaciones de diversas partes del mundo. Localizaron 153 artículos, donde se analiza el número de artículos publicados por año, producción por revista, el índice de autoría y los contenidos de los trabajos. Los resultados muestran que el periodo de mayor producción es 2001 a 2006 , con un total del $36 \%$; la revista que más ha publicado en el tema es la Journals of Consulting and Clinical Psychology con el 12.4\%. Asimismo, los artículos se encuentran firmados por uno y dos autores, y los de mayor productividad son David A. Cole (University of Notre Dame, USA) y Victoria Del Bario (UNED, Universidad Nacional de Educación a Distancia, España). El instrumento más utilizado es el CDI (Children's Depression Inventory), con el 46.1\%. Finalmente, los contenidos más comunes corresponden a los estudios de confiabilidad y validez de instrumentos de medición y las revisiones teóricas. 5

En el 2015, se hace público los resultados del Análisis Bibliométrico de la Depresión Infantil de Restrepo \& Alarcón, el objetivo de este estudio fue identificar las tendencias en la investigación de la depresión infantil mediante un análisis bibliométrico de artículos publicados entre enero de 2010 y enero de 2015, en las bases de datos EBSCO, Scopus y Scielo. Se incluyeron 146 artículos, escritos en inglés, español y portugués. En el trabajo se identifican los autores más productivos y las tendencias de los autores con respecto al sexo, profesión y producción; así como los países, instituciones y revistas que más publicaron sobre dicho trastorno. La edad y la ansiedad son las variables que con más frecuencia se asocian al tema; de igual forma, se identificó el Children Depression Inventory (CDI) como el instrumento más común para medir la depresión infantil. En este periodo de tiempo destacan los artículos centrados en investigaciones sobre poblaciones con diferentes estados de salud, diseños correlacionales, de corte transversal y escritos por múltiples autores. Al analizar los resultados obtenidos se observa un creciente interés y preocupación de los investigadores por conocer la frecuencia de los trastornos del estado de ánimo y más específicamente de la depresión en niños, y la asociación e influencia que esta puede tener para su desarrollo.6 Buitrago, L. Laverde, A.; Álvarez, A.; Estupiñan. L también realizaron una investigación con el objetivo principal de analizar la producción científica publicada entre 2008 y 2013 sobre suicidio en niños, niñas y adolescentes mediante un estudio bibliométrico de tipología macro con tres categorías de análisis de indicadores, a saber: científicos, de colaboración, impacto, y actividad. Investigación documental de tipo bibliométrico con base metodológica de análisis sistémico de información de carácter retrospectivo. Los resultados de esta investigación se registraron de la siguiente manera: el análisis descriptivo de los datos en los cuales se encontraron 57 artículos y se seleccionaron 28 artículos equivalentes al $49 \%$, la producción por años de las publicaciones fue mayor en el 2012 con un $25 \%$, el $86 \%$ de los artículos se encuentran en inglés, se evidencia que el reporte de suicidios consumado se ha presentado en los dos géneros, la metodología utilizada en la muestra de los 28 artículos analizados es de tipo correlacional, el tipo de documento de los artículos revisados corresponde a 23 artículos de revisión junto con un inventario de factores de riesgo individuales, asociados con la conducta suicida en niños, niñas y adolescentes7. El Estado Peruano no es ajeno a la problemática de la salud mental de niños y adolescentes, es así que para el quinquenio 2010-2014 la estableció como una de las prioridades de investigación a nivel nacional, y con 
propósito de establecer una línea base en la producción científica y poder orientar la temática de futuras investigaciones es que nos motivamos a generar este estudio bibliométrico.

\section{OBJETIVO}

Analizar la producción científica publicada entre 2003 y 2014 sobre depresión y suicidio en niños, niñas y adolescentes mediante un estudio bibliométrico.

\section{MATERIAL Y METODOS}

Esta investigación se define como una investigación documental bajo la técnica de análisis bibliométrico. La bibliometría parte de la información bibliográfica de publicaciones científicas para crear indicadores, con un análisis tipo Ex post o de resultados. El diseño de este estudio es de carácter retrospectivo se seleccionaron 94 artículos científicos publicados en lengua español, tanto de Latinoamérica como Europa.

El procedimiento seguido fue: se realizó una búsqueda de artículos, comprendidos entre los años 2003 al 2014, de las bases de datos Hinari (Organización Mundial de la Salud) y Lilacs, Redalyc, Medline, Scielo, Psicothema con los descriptores depresión niños, depresión adolescentes, suicidio niños, suicidio adolescentes.

Al ubicar y constatar que cada artículo hiciera referencia a la temática de la depresión y suicidio en niños y adolescentes, se procedió a realizar una base de datos para analizar cada una de las variables a tener en cuenta en el análisis bibliométrico, para ello se generó una Ficha Bibliométrico ad hoc (Jesús, 2015). En éste las variables incluidas fueron: Tema de la investigación, país, año de publicación, autor(es, tipo de investigación, diseño de investigación, Caracterización de la muestra: tipo, tamaño, genero, edades, nivel socioeconómico, nivel educativo. Instrumentos más utilizados, distribución de trabajos por número de firmas,

Una vez organizada la información, se efectuó el análisis de frecuencia y porcentual para lograr obtener los datos más relevantes sobre el estado de la investigación.

\section{RESULTADOS}

Tabla 1. Temas de investigación periodo 2003-2014

\begin{tabular}{lcc}
\hline & f & \% \\
\hline DEPRESION EN NIÑOS & 20 & 21.2 \\
DEPRESION EN ADOLESCENTES & 22 & 23.4 \\
RIESGO SUICIDA EN NINOS & 1 & 1.1 \\
RIESGO SUICIDA ADOLESCENTES & 3 & 3.2 \\
IDEACION SUICIDA NINOS & 1 & 1.1 \\
IDEACION SUICIDA ADOLESCENTES & 6 & 6.4 \\
INTENTO SUICIDA EN NINOS & 0 & 0.0 \\
INTENTO SUICIDA EN ADOLESCENTES & 6 & 6.4 \\
INTENTO SUICIDA EN NINOS Y ADOLESCENTES & 2 & 2.1 \\
SUICIDIO EN NIÑOS & 1 & 1.1 \\
SUICIDIO EN ADOLESCENTES & 6 & 6.4 \\
DEPRESION Y SUICIDIO EN NIÑOS & 0 & 0.0 \\
DEPRESION Y SUICIDIO EN ADOLESCENTES & 3 & 3.2 \\
SUICIDIO EN NIÑOS Y ADOLESCENTES & 1 & 1.1 \\
CONDUCTAS SUICIDA EN NIÑOS Y ADOLESCENTES & 1 & 1.1 \\
DEPRESION,IDEACION E INTENTO SUICIDA EN & & \\
ADOLESCENTES & 1 & 1.1 \\
DEPRESION EN NIÑOS Y ADOLESCENTES & 17 & 18.1 \\
DEPRESION Y SUICIDIO EN NIÑOS Y ADOLESCENTES & 3 & 3.2 \\
\hline & 94 & 100
\end{tabular}


Tabla 2. Producción científica por países hispanohablantes

\begin{tabular}{lcc}
\hline PAIS & $\mathrm{f}$ & $\%$ \\
\hline Argentina & 1 & 1.2 \\
Bolivia & 1 & 1.2 \\
Colombia & 25 & 26.6 \\
Cuba & 3 & 3.2 \\
Chile & 13 & 13.8 \\
Ecuador & 0 & 0.0 \\
Guatemala & 1 & 1.2 \\
Puerto Rico & 3 & 3.2 \\
Perú & 13 & 13.8 \\
Paraguay & 1 & 1.2 \\
Venezuela & 1 & 1.2 \\
México & 17 & 17.02 \\
España & 15 & 15.9 \\
\hline TOTAL & 94 & 100
\end{tabular}

Figura 1.- Producción científica por años.

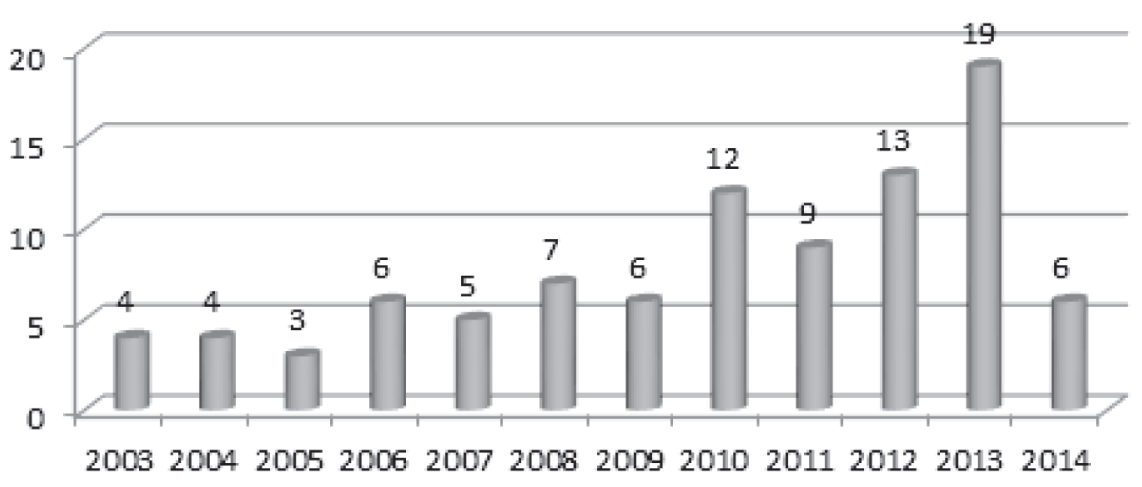

Figura 2. Tipos de investigación.
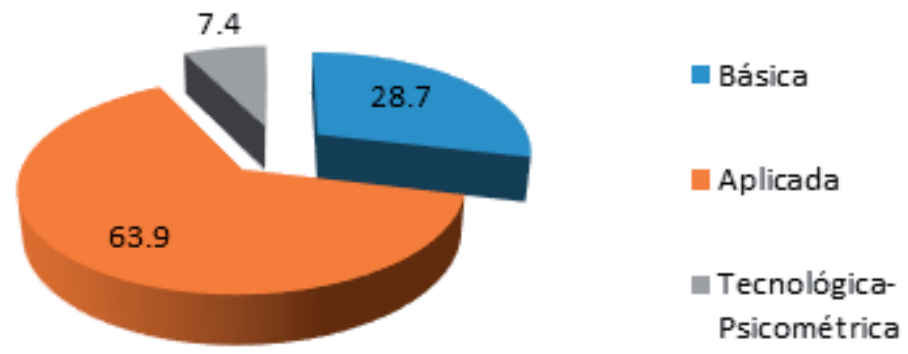
Tabla 3: Diseño de investigación en las Investigaciones aplicadas.

\begin{tabular}{lcc}
\hline DISENO DE INVESTIGACION & $\mathrm{f}$ & $\%$ \\
\hline Estudio de caso & 3 & 4.5 \\
Descriptivo simple & 11 & 16.4 \\
Descriptivo comparativo & 9 & 13.4 \\
Descriptivo correlacional & 15 & 22.4 \\
Descriptivo causal-comparativo & 0 & 0.0 \\
Transversal & 20 & 29.8 \\
Longitudinal & 4 & 6.0 \\
Experimental & 2 & 3.0 \\
otros & 3 & 4.5 \\
\hline TOTAL & 67 & 100
\end{tabular}

Figura 3: Caracterización de la muestra según tipo de muestreo

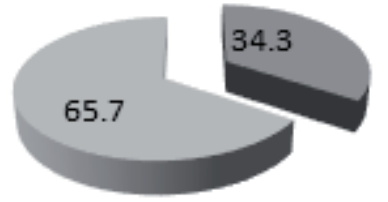

$$
\begin{aligned}
& \text { W Probabilístico } \\
& \text { No Probabilística }
\end{aligned}
$$

Tabla 4: Caracterización de la muestra según tamaño

\begin{tabular}{lcc}
\hline TAMANO DE LA MUESTRA & $\mathrm{f}$ & $\%$ \\
\hline Menores a 50 & 8 & 11.9 \\
Entre 51 y 100 & 7 & 10.4 \\
Entre 101 y 500 & 27 & 40.3 \\
Entre 501 y 1000. & 16 & 24 \\
Más de 1000 & 9 & 13.4 \\
\hline TOTAL & 67 & 100 \\
\hline
\end{tabular}

Figura 4: Caracterización de la muestra según género
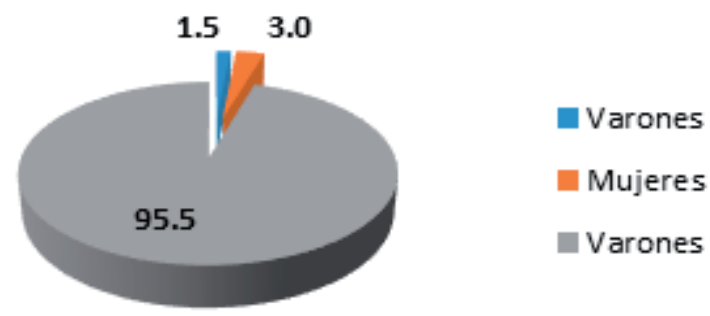

Varones y mujeres

Tabla 5. Caracterización de la muestra según edades.

\begin{tabular}{ccc}
\hline \multicolumn{2}{c}{ EDADES DE LA } & f \\
MUESTRA & 12 & $\%$ \\
\hline Niños (5 - 9 años) & 18 & 26.0 \\
Adolescentes (10-14 años) & 31 & 46.3 \\
Adolescentes (15-19 años) & 6 & 8.9 \\
No precisa & 67 & 100 \\
\hline TOTAL
\end{tabular}


Tabla 6. Caracterización de la muestra según nivel socioeconómico

\begin{tabular}{|c|c|c|}
\hline NIVELES SOCIOECONÓMICO & $\mathrm{f}$ & $\%$ \\
\hline Alto & 0 & 0.0 \\
\hline Medio & 8 & 11.9 \\
\hline Bajo & 0 & 0.0 \\
\hline Extrema pobreza & 0 & 0.0 \\
\hline No precisa & 59 & 88.05 \\
\hline TOTAL & 67 & 100.0 \\
\hline
\end{tabular}

Tabla 7. Caracterización de la muestra según nivel educativo

\begin{tabular}{lcc}
\hline NIVEL EDUCATIVO & $\mathrm{f}$ & $\%$ \\
\hline Primaria & 12 & 17.9 \\
Secundaria & 45 & 67.2 \\
Bachillerato (Centro Pre Universitario) & 2 & 2.9 \\
Universidad (1 año) & 2 & 2.9 \\
No precisa & 6 & 9 \\
\hline \multicolumn{1}{c}{ TOTAL } & 67 & 99.9 \\
\hline
\end{tabular}

Figura 5. Instrumentos de medición

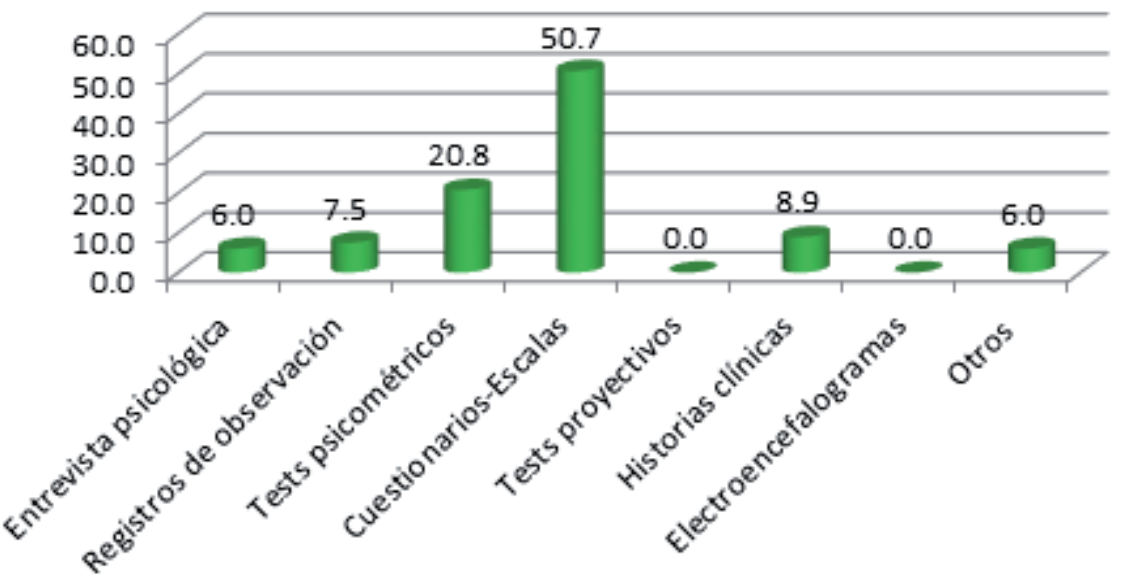

Tabla 8. Instrumentos psicológicos más utilizados

Cuestionario de depresión para niños CDS de Lang y Tisser 1983 adaptación española

Escala del centro de estudios epidemiológicos de depresión CES-D (Radlof,1997).

Inventario de depresión de niños (CDI, Kovacs 1992, 1997).

Escala de ideación suicida (Roberts, 1980).

Inventario de ideación suicida positiva y negativa de PANSI, 1998 adaptado

Escala de ideación suicida de Beck.

Cuestionario de salud y bienestar niños (as) adolescentes, 2002 KIDSCREEM

Cuestionario para el diagnóstico de los cuadros depresivos de Calderón.

Child Depression Seace (CAS, Tisher y Long 1979).

Cuestionario de ideación suicida.

Escala de riesgo suicida SPS.

Cuestionario de las manifestaciones depresiva- Alonso Fernández.

La RCADS-30(Escala de ansiedad, depresión infantil).

Inventario de depresión infantil. 
Figura 6. Distribución de trabajos por número de firmas

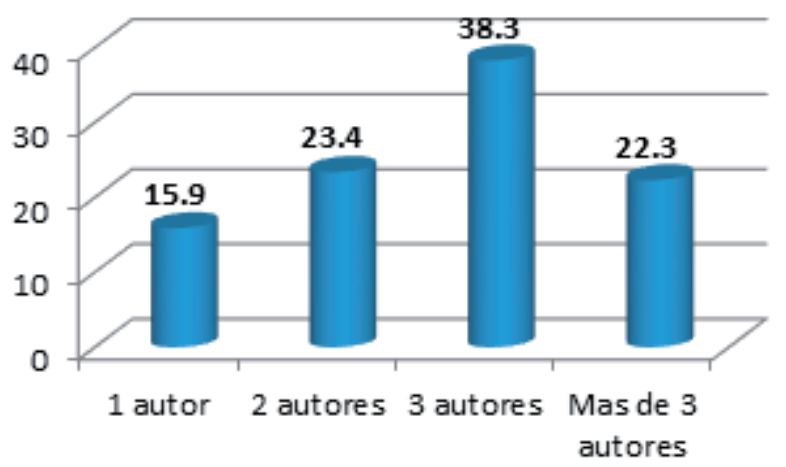

\section{DISCUSION}

En pleno siglo XXI ya no cabe duda que la depresión y suicidio en nuestros niños, niñas y adolescentes es un problema de salud pública que preocupa a la sociedad en general, las proyecciones de la Organización Mundial de la Salud, sobre la salud mental en la población infanto-juvenil no son alentadoras por ello, considerando que es importante conocer el estado de la producción científica en este tema y ser un referente para posteriores investigaciones se realizó el presente estudio de investigación.

Producto del análisis bibliométrico de las investigaciones en depresión y suicidio en niños y adolescentes periodo 2003 y 2014, se encuentra que el tema más investigados es depresión en niños, seguidos de depresión en adolescentes y de depresión en niños y adolescentes, siendo todavía bajo el interés por temas como ideación e intento suicida y más aún depresión y suicidio en niños y adolescentes.

Se hace necesario indagar en las conductas relacionadas entre depresión y el acto suicida, de modo que conociendo por ejemplo datos sobre ideación suicida, riesgo suicida e intentos suicidas, las estrategias de prevención e intervención oportuna pudieran ser más eficaces.

Los países con más producción científica como Colombia, México y España, estaría reflejando el nivel de sensibilización y compromisos de instituciones académicos y profesionales con el tema de la depresión y suicidio en su población infanto juvenil. Posiblemente son aquellos donde las políticas de salud mental están mejor orientados desde el rubro de la prevención y promoción así como con las estrategias de tratamiento o recuperación.

Se aprecia que la producción científica en el tema de la depresión en niños ya adolescentes empieza a incrementarse a partir del año 2012, datos que coincide con la afirmación de Méndez, al señalar que el tema está cada vez más concitando la atención de más académicos y especialista así como con los hallazgos de Buitrago 7

El tipo de investigación es la aplicada seguidos de la investigación básica, lo que refleja que los investigadores están más preocupados por poner en ventana la situación real o problemática de grupos infanto-juveniles respecto a la depresión y suicidio, siendo el diseño de investigación más utilizado el descriptivo correlacional, ello nos está señalando que se está optando por investigaciones multivariables, que utilizando el análisis factorial nos va a permitir establecer con mayor precisión el grado de relación e influencia mutua entre variables asociadas con la depresión o suicidio, de esta forma se estaría dando más evidencias a favor de las definiciones que señalan que la depresión y suicidio son entes complejos y multicausales, sin duda, este tipo de investigaciones; nos ayudarán a orientar mejor las estrategias de prevención, promoción e intervención. Sin embargo, consideramos que no se debe dejar de lado las investigaciones teóricas; pues éstas por su naturaleza son importantes espacios de reflexión y crítica para valorar o juzgar modelos explicativos o de intervención. Estos datos considerando los hallazgos de Diez3 y Palacios4 son una evidencia de que si en los años pasados las investigaciones teóricas eran las más preponderantes ahora lo son las investigaciones aplicadas, que por cierto coinciden con los hallazgos de Restrepo 6.

En lo referente a las muestras, el tipo de muestreo preponderante es el no probabilístico intencional, siendo el tamaño de las muestra entre 101 y 500, lo que refleja que aún es un reto asumir por investigaciones a gran escala que nos den las fuerza estadística para generalizar resultados, son aún los escasos los países donde se ha optado por un censo nacional de la salud mental. 
Encontramos que las investigaciones se han realizado con muestras de ambos géneros (95.5\%)-datos coincidente con los de Buitrago 7que con muestras exclusivamente de un género, esto nos permite suponer que la sintomatología depresiva no diferencia entre ambos, sin embargo queda pendiente asegurar con evidencias que no existe diferencias significativas entre la depresión de un niño o niña, un adolescente y una adolescente. Creemos que en este sentido las investigaciones cualitativas pueden enriquecer información para una mayor y mejor comprensión de estos temas.

Los adolescentes del nivel secundario son los más investigados (73\%) frente al $18 \%$ de niños, esto se deba probablemente a que son los casos que más se han reportado o también a la disponibilidad a responder a los diferentes instrumentos de medición. Queda como reto seguir generando estrategias metodológicas para investigar la depresión y riesgo suicida en los niños.

Si bien es cierto la depresión y riesgo suicida no discrimina clase social, nos llama la atención que el $88 \%$ no reporta el nivel socioeconómico de las muestras, los que lo hicieron señalan clase media (7\%), sin embargo, creemos que aún queda pendiente ampliar y/o profundizar las características de la depresión según estratos sociales, de hecho los resultados van a permitir a los profesionales comprender un problema psicológico desde una mirada social y cultural y de hecho las estrategias de evaluación e intervención serán más pertinente.

Entre los instrumentos más utilizados se reportan los cuestionarios y escalas y tests psicométricos siendo el Cuestionario de depresión para niños CDS el más utilizado. Ello permite confiar en la objetividad de los resultados toda vez que son instrumentos que demuestran grados significativos de validez y confiabilidad y además por la factibilidad de su administración. Sin embargo, considerando que muchos de los instrumentos son generados en otros contextos geográficos, es un imperativo para los investigadores generar estudios de adaptación o baremación de estos instrumentos psicológicos, sobre todo si se considera que los tests psicométricos están expuestos a la influencia de variables sociodemográficas o en su defecto construir escalas cuestionarios concordantes a su realidad.

Finalmente, se encuentra que más del $84 \%$ de las publicaciones corresponde a autorías múltiples (más de dos autores). Esto es una evidencia a favor de las ventajas del trabajo en equipo, considerando que la mayoría de ellos son de la misma disciplina creo que se hace ne- cesario promover las investigaciones transculturales y multidisciplinarias.

\section{CONCLUSIONES}

- Los temas más investigados son depresión en niños $(21.2 \%)$, seguidos de depresión en adolescentes $(23.4 \%)$.

- Los países que más han producido son Colombia (26.6\%), seguidos de México (17.02\%) y España $(15.9 \%)$.

- La producción científica empieza a incrementarse a partir del año 2012 en adelante.

- Las investigaciones de tipo aplicada-sustantiva $(63.9 \%)$ se imponen sobre las investigaciones puras $(28.7 \%)$

- Sobresale el Diseño descriptivo correlacional (22.4\%) y el descriptivo simple (16.4\%).

- Las muestras suelen ser del tipo no probabilístico intencional (65.7\%) siendo el tamaño mayoritario de 101 y 500 participantes (40.3\%), en ellas se han incluido varones y mujeres (95.5\%), siendo los adolescentes del nivel secundario los más investigados (73\%), llama la atención que el $88 \%$ no reporta nivel socioeconómico.

- Entre los instrumentos más utilizados se reportan los Cuestionarios y Escalas y tests psicométricos 71\%) siendo el Cuestionario de depresión para niños CDS el más utilizado.

- Más del $84 \%$ de producción corresponde a autorías múltiples.

\section{REFERENCIAS BIBLIOGRÁFICAS}

1. Organización Mundial de la Salud (OMS), Informe mundial sobre la salud adolescente Health for the World's Adolescents, Disponible en Disponible en: http://www.who.int/mediacentre/news/releases/2014/focus-adolescent-health/es/.

2. Buitrago, L. Laverde, A.; Álvarez, A.; Estupiñan. L. .Análisis de la producción científica publicada entre 2008 y 2013 sobre suicidio en niños, niñas y adolescentes mediante un estudio bibliométrico. En Revista del Hospital Psiquiátrico de La Habana Volumen 12 nro. 2-2015. Disponible en http://www.revistahph.sld.cu/2015/Nro\%202/analisis\%20de $\% 20$ la\%20produccion.html.

3. Diez, M. Análisis bibliométrico sobre depresión infantil en España. En revista internacional de Psicología Clínica y de la Salud. Vol. 3 nro.1 pp. 645653. 2003 Disponible en http://www.redalyc.org/ 
pdf/337/33730313.pdf

4. Palacios-Espinosa, X.; Barrera, A, y Ordóñez, M. Y Peña, M . Análisis bibliométrico de la producción científica sobre suicidio en niños en el período 1985-2005 . En Avances en psicología latinoamericana Bogotá . Vol. 25(2). Pp.40-62. 2007. Disponible en file:

//C:/Users/user/Downloads/An\%C3\%A1lisis\%20bibliom $\%$ C3\%A9trico $\% 20 \mathrm{de} \% 201 \mathrm{a} \% 20$ producci $\%$ C3\%B3n\%20cient $\%$ C3\%ADfica $\% 20$ sobre $\% 20$ suicidio $\% 20$ en $\% 20$ ni $\%$ C3\%B1 os $\% 20$ en $\% 20$ el $\% 20$ per\%C3\%ADodo\%201985-2005.pdf

5. Herrera, Eduar.; Núñez, A.Tobón, S.;Arias, D. Análisis bibliométrico de la depresión infantil En pensamiento psicológico. Vol 5, Nro. 12, 2009. Pp. 59-70. Disponible en file:

//C:/Users/user/Downloads/An\%C3\%A1lisis\%20 bibliom $\%$ C3\%A9trico\%20de\%20la\%20depresi $\%$ C3\%B3n\%20infantil.pdf

6. Restrepo. M. \& Alarcón, M. Análisis Bibliométrico de la Depresión Infantil. Tesis para optar el Titulo de Psicóloga. Universidad del Rosario. Bogotá.2015. Disponible en http://repository.urosario.edu.co/ bitstream/handle/10336/10657/1020777461-2015. pdf? sequence $=1$

Fecha de recepción: 02/04/15

Fecha de aprobación: 06/05/15

Correspondencia: Mercedes Jesús Peña

Email:mjesus@mail.upla.edu.pe 